\title{
Intrinsic Intraday Variability in the Gravitational Lens System B0218+357
}

\author{
Andrew Biggs, Ian Browne, Peter Wilkinson \\ University of Manchester, Jodrell Bank Observatory
}

\begin{abstract}
Radio monitoring of the gravitational lens system B0218+357 reveals it to be a highly variable source with variations on timescales of a few days correlated in both images. This shows that the variability is intrinsic to the background lensed source and suggests that similar variations in other intraday variable sources can also be intrinsic in origin.
\end{abstract}

\section{Introduction}

The origin of the radio-frequency intraday variability (IDV) seen in many flatspectrum sources is a major astrophysical puzzle, particularly the extremely high brightness temperatures that are implied by the very short timescale of the variations. If these are intrinsic to the source then the implied source size is extremely small which leads to brightness temperatures that can be as high as $10^{18} \mathrm{~K}$ e.g. PKS 0405-385 (Kedziora-Chudczer et al. 1997). Brightness temperatures as high as these are difficult to reconcile with the inverse Compton limit of $\sim 10^{12} \mathrm{~K}$ for incoherent synchrotron sources without assuming improbable Doppler factors of $\sim 100$ for the emitting regions. An alternative to an intrinsic origin for the observed variations is that they arise due to a propagation effect such as interstellar scintillation (Rickett 1990). Scintillation can, however, only account for variations at radio wavelengths.

\section{VLA Monitoring of $\mathrm{B} 0128+357$}

Even though both intrinsic and extrinsic explanations have their difficulties, it would represent considerable progress if it could be proven that the variations had one or the other origin. The study of radio sources which have multiple gravitational images offers an elegant way to do this. If an IDV source is multiply imaged, only intrinsic variations should be correlated in the images. We have recently measured a time delay of $10.5 \pm 0.4 \mathrm{~d}$ in the lensed system B0218+357, exploiting its rapid radio variability (Biggs et al. 1999). We show the VLA $15-\mathrm{GHz}$ light curves in Fig. 1.

The variability events in the light curves correspond to $T_{\mathrm{b}}$ in the range $10^{14}-10^{15} \mathrm{~K}$ for the source at $z=0.96$ and with the lens magnifications taken into account. If we invoke Doppler boosting of a relativistic jet directed close to our line of sight, these brightness temperatures can be brought below the Compton limit with moderate Doppler factors of $\sim 10$. However, there has never been a detection of superluminal motion (or any structural change for that 

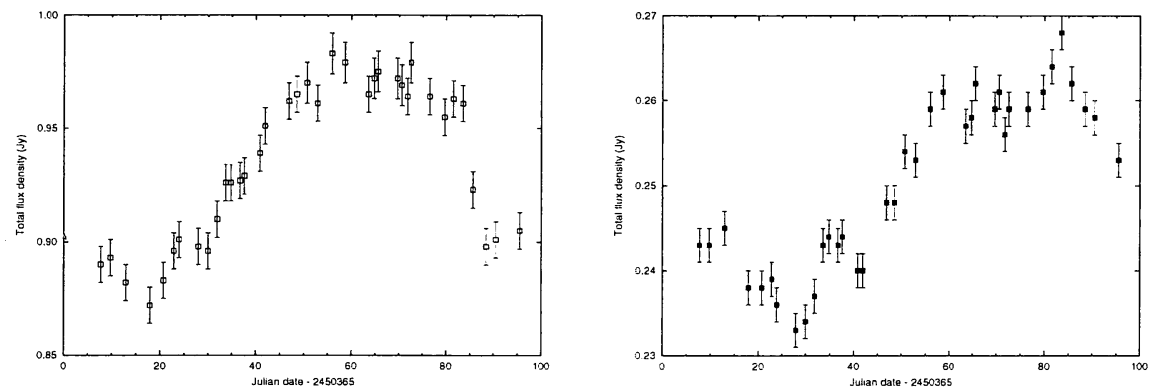

Figure 1. VLA $15 \mathrm{GHz}$ monitoring lightcurves of component A (left) and $B$ (right).

matter) in the radio core of B0218+357, despite regular multi-frequency VLBI observations over the past ten years.

\section{Discussion}

Could the correlated variability seen in the images of $0218+357$ be produced by any of the extrinsic mechanisms? First let us consider interstellar scintillation. Let us assume that a typical velocity of the local Galactic ISM with respect to the Earth is $20 \mathrm{~km} \mathrm{~s}^{-1}$. To produce correlated changes in A and B with a time lag of $10 \mathrm{~d}$ the screen would have to move $\geq 335$ mas in this time and be at a distance of less than $0.3 \mathrm{pc}$. Since this is very much less than the scale height of the Galactic disk we can rule out the scintillation explanation.

\section{Conclusions}

The source in the lens system B0218+357 is an intraday variable and the variations on a timescale of a few days are correlated in both images. Thus on these timescales, at least, the mechanism producing the major variations must be intrinsic and any contribution from scintillation small.

Acknowledgments. The VLA is operated by the National Radio Astronomy Observatory, which is a facility of the National Science Foundation operated under cooperative agreement by Associated Universities, Inc. This research was supported in part by European Commission, TMR Programme, Research Network Contract ERBFMRXCT96-0034 "CERES".

\section{References}

Biggs, A. D., Browne, I. W. A., Helbig, P., Koopmans, L. V. E., Wilkinson, P. N., Perley R. A., 1999, MNRAS, 304, 349

Kedziora-Chudczer, et al., 1997, ApJ, 490, L9

Rickett, B. J., 1990, ARA\&A, 28, 561 